\title{
Water line intensities in the near-infrared and visible
}

\author{
Roman N Tolchenov, ${ }^{\text {a }}$ Mizuho Tanaka, ${ }^{\text {a }}$ Jonathan Tennyson,,${ }^{\text {a,1 }}$ \\ Nikolai F Zobov, ${ }^{a b}$ Sergei V Shirin, ${ }^{a}$ b Oleg L Polyansky, ${ }^{a b}$ \\ Ahilleas N Maurellis ${ }^{c}$ \\ ${ }^{\mathrm{a}}$ Department of Physics and Astronomy, University College London, London, \\ WC1E $6 B T, U K$ \\ ${ }^{\mathrm{b}}$ Permanent address: Institute of Applied Physics, Russian Academy of Science, \\ Uljanov Street 46, Nizhnii Novgorod, Russia 603024. \\ ${ }^{\mathrm{c}}$ SRON National Institute for Space Research, Sorbonnelaan 2, 3584 CA Utrecht, \\ The Netherlands.
}

\begin{abstract}
Water is the single most important molecule for models of the earth's atmosphere but line parameters for water, particularly at shorter wavelengths, are difficult to measure reliably. We suggest that the most reliable way of generating water line parameters is to combine data obtained from a variety of sources, thereby separating line parameter determination into results for strong lines, for weak lines and for isotopically-substituted water. Theoretical considerations which are addressed include line assignments and labeling of energy levels and the prospects of a full theoretical solution to the water vapor problem. Particular attention is paid to strong line absorption intensities in the near infrared where recent studies have given significantly different results. The experimental data used to construct the ESA-WVR linelist (Schermaul et al, J. Molec. Spectrosc., 208, 32 (2001)) is re-analyzed with a focus on effects due to pressure determination in the cell, subtraction of the baseline and parameterization of the line profiles. A preliminary re-analysis suggests that the line intensities given by the ESA-WVR study should be closer to those of Brown et al (J. Molec. Spectrosc., 212, 57 (2002)) used in the HITRAN. This shows the vital importance of validating the data for water by independent means.
\end{abstract}

Key words: Water vapor; Line parameters; Atmospheric radiation. $P A C S$ :

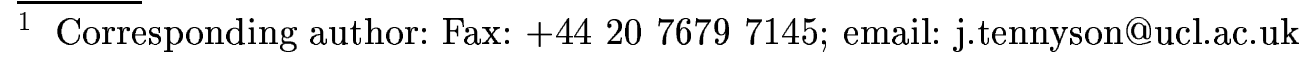

Preprint submitted to Elsevier Science

24 March 2003 


\section{Introduction}

Water is molecule number one in the list of atmospheric species in the HITRAN database [1,2]. Its number one status is a reflection of the dominant influence water has in both the absorption of sunlight as it passes through our atmosphere and its role as the predominant greenhouse gas. The rotationvibration spectrum of water is not only complicated but also extensive. It is therefore unsurprising that there have been many detailed studies of the water spectrum, see refs [3-18] for examples at the near-infrared and visible wavelengths which concern us here. Despite these studies, even basic line parameters. such the intensity of the strongest lines in the near-infrared and optical region, remain a subject of debate [19].

In particular the influence of relatively small changes $(\sim 10 \%)$ in line intensities for water have been shown to have significant influence on radiation transfer in the earth's atmosphere. Indeed changes of this magnitude could account for much of the missing absorption in the atmosphere [20,21]. Recently Belmiloud et al [19] suggested that the data in the 1996 edition of HITRAN [1], and those corrected by Giver et al [22], systematically underestimate the effects of strong water lines in the near infrared and red region of the spectrum, among the most critical regions for these absorptions. The details of the experiment giving rise to this assertion and the associated parameters were subsequently published by Schermaul et al [14,23] and made available electronically as the ESA-WVR linelist [24]. The ESA-WVR linelist covered the range 8592 to $15000 \mathrm{~cm}^{-1}$. In a parallel study Brown et al [16] analysed in detail the line parameters of water in the range 10150 to $11190 \mathrm{~cm}^{-1}$. Their complete data, which covered the extended range $9650-11190 \mathrm{~cm}^{-1}$, is included in the 2000 edition of HITRAN. Brown et al's data also leads to an increase in the overall absorption by water but only by about half that given by ESAWVR. Brown et al's analysis also emphasized the difficulty of getting reliable data for absorption by isotopically-substituted water from spectra recorded for water in natural abundance. Coheur et al [17] have also recently reported a long pathlength study of water vapor and water-air spectra which covers the 13000 to $26000 \mathrm{~cm}^{-1}$ region. This work again finds increased absorption by water vapor, by about $5 \%$, compared to HITRAN but, in the region where they overlap, somewhat lower intensities than given by ESA-WVR [24]

In this paper we attempt to develop a strategy for determining reliable line intensities for water. This strategy addresses strong lines, weak lines, isotopicallysubstituted water and spectral assignments. For the strong line data we reanalyze the data recorded as part of the ESA-WVR study to try and pinpoint the source of the discrepancy between that work and the study of Brown et al. Finally the need for independent means of testing the reliability of water vapor line parameters is emphasized. 


\section{Data sources: an overview}

The large dynamic range of water absorption intensities required for atmospheric modeling means that line parameters are difficult, if not impossible, to determine from a single experiment. To accumulate the best possible data for each case, the most reliable way of generating water line parameters would therefore appear to be to combine data obtained from a variety of sources.

The wavelengths of strong line absorptions by water in the near-infrared and optical are well known $[3,4,12,13]$. However other line parameters, particularly the absorption intensities, can be difficult to determine [19]. Schermaul et al [14] performed a series of pure water vapor and water-air experiments in the range $8600-15000 \mathrm{~cm}^{-1}$. These experiments were specifically aimed at accurately determining the absorption intensities for the strong lines. The experiments were performed using pathlengths varying from $5 \mathrm{~m}$ to $512 \mathrm{~m}$ in order to counter saturation effects. Although an extensive analysis of their water-air experiments has been reported [14,23], it would appear that their pure water vapor data has not previously been analyzed.

Brown et al [16] recently reported a careful study which covered a smaller wavelength region centered on $0.94 \mu \mathrm{m}$ (specifically 10150 to $11190 \mathrm{~cm}^{-1}$ ). The intensities obtained by Schermaul et al [14] were significantly $(6-25 \%)$ stronger than previous studies $[1,4,6,22]$. While a combination of both alternative analysis and $a b$ initio theory supports Schermaul et al's results [19], the intensities reported by Brown et al [16] are consistently smaller than those of Schermaul et al for the same region, although they are also somewhat larger than the previous values given in HITRAN [1]. Reasons for this differences are explored in detail in the next section.

Schermaul et al combined experimental data on strong line parameters with estimates of the position and strength of the weaker lines taken from variational nuclear motion calculations [25] to give a database, ESA-WVR [24], for the $8600-15000 \mathrm{~cm}^{-1}$ region [23]. Although this approach has some merit in terms of completeness, theory is not yet sufficently advanced for this approach to be accurate, see ref. [26] for example. An alternative approach is therefore needed to obtain reliable weak line parameters.

While water-air spectra recorded at atmospheric pressures provide the best way of determining parameters for strong water lines, the greater sensitivity given in (low pressure) pure water vapor spectra provide a much more reliable way of obtaining information on weak water lines. Schermaul et al [15] recorded a series of spectra of pure water vapor using long pathlengths (up to $800 \mathrm{~m}$ ) and long integration times. These spectra have been analyzed using variational nuclear motion calculations $[15,27,28]$. A feature of all of 
these studies, and other long pathlength weak line spectra recorded at shorter wavelengths $[12,13,17,29]$, is the presence of new weak water lines which had not been observed in previous studies and are therefore absent from database compilations. Theory suggests [30] that there are new water transitions to be observed at essentially all levels of sensitivity. Model atmosphere calculations [20] have demonstrated that inclusion of these lines gives a significant, $\sim 3$ $\mathrm{Wm}^{-2}$, effect on the absorption by the earth's atmosphere.

$\mathrm{H}_{2}{ }^{18} \mathrm{O}$ is thought to be the fifth most important absorber of sunlight in the Earth's atmosphere. Long pathlength spectra, such as those of Schermaul et $a l$ [15] and Brown et al [16], show features which can be attributed to $\mathrm{H}_{2}{ }^{18} \mathrm{O}$, and possibly even less abundant isotopomers. However this is not the best way of determining line parameters for these trace species. Almost twenty years ago Chevilliard and co-workers [5,31,32] recorded a series of long-pathlength spectra using samples which were isotopically enhanced for $\mathrm{H}_{2}{ }^{18} \mathrm{O}$ and $\mathrm{H}_{2}{ }^{17} \mathrm{O}$. It is only recently that these spectra have been analyzed at the frequencies near the maximum in the solar spectrum [33-35]. These spectra clearly provide the best source of data for the trace isotopomers of water.

Variational nuclear motion calculations have led to a major improvement in the theoretical treatment of water spectra [36]. Although fully ab initio calculations are still not as accurate as measurements [37], they can play an important role in resolving problems with water spectra. In particular most recent studies of water rotation-vibration spectra in the optical have relied on variational nuclear motion calculations to assign quantum numbers to individual lines $[12,13,15,27-29,34,35,25,38-40]$. Indeed these methods have been able to resolve a number of problems with previous assignments, particular those concerning the correct identification of high-lying vibrational states [12,13]. Variational calculations may not be accurate but they are complete. This makes them useful for looking at systematic errors in experimental data caused, for example, by cut-offs due to sensitivity [30]. Finally ab initio variational calculations can provide an independent means of calculating line intensities [26]. There is likely to be increasing emphasis on this activity as dipole surfaces for water are systematically improved $[25,41,42]$ and their accuracy become competitive with, or even better than, experiment.

One problem when compiling information on the rotation-vibration spectrum of water from different sources is validating the data. It is standard practice to validate line profile fits against the experimental data from which they originate. However as illustrated below, this method often fails to identify systematic errors. 


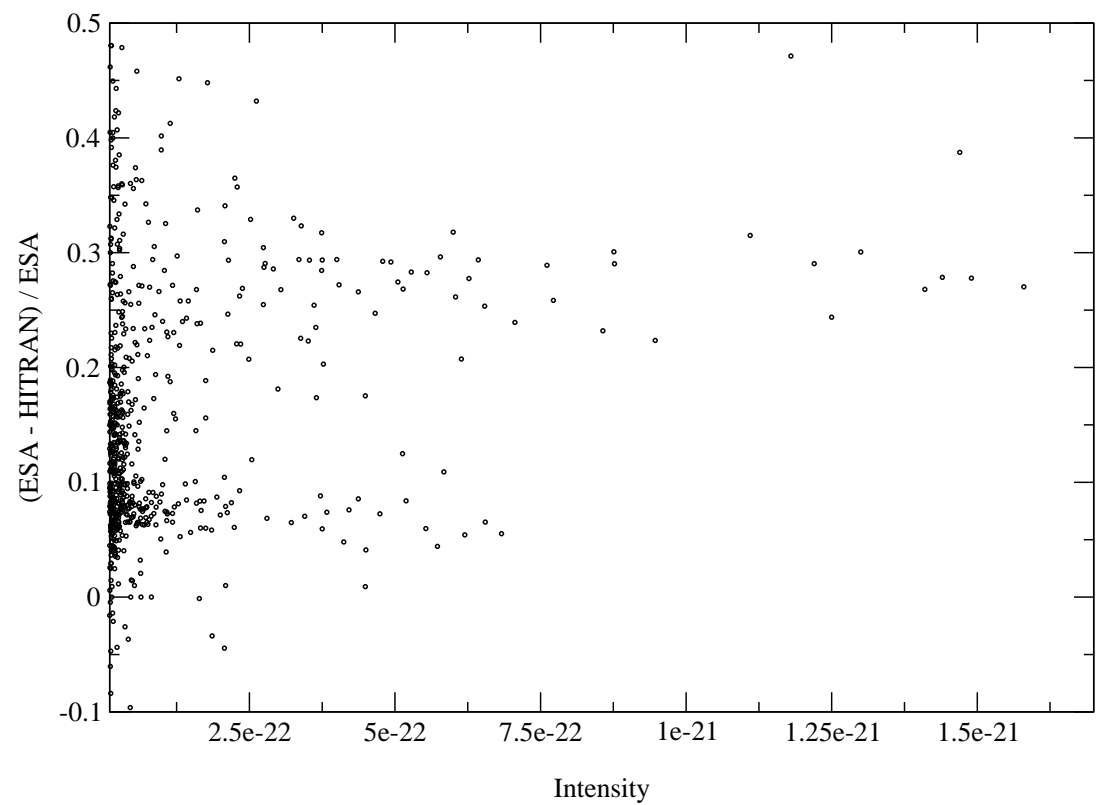

Fig. 1. Relative differences in line intensity for strong water vapor lines obtained from the ESA-WVR database [24] and HITRAN [2] as a function of ESA-WVR line intensity (in cm molecules ${ }^{-1}$ ) in the range $8600-15000 \mathrm{~cm}^{-1}$. Clear systematic differences occur at approximately the $8 \%$ and $28 \%$ levels which may be linked to the experimental source of the line data (see text).

\section{Re-analysis of the strong line intensities}

Figure 1 compares line intensities reported in the ESA-WVR [24] and HITRAN [2] databases. The figure shows clear systematic differences. A frequencydependent analysis shows that the data reported by Brown et al [16] largely gives a linear feature showing systematic differences at the less than $10 \%$ level, while larger systematic differences arise from longer wavelength spectra. Brown et al made a particularly careful study and obtained results which differ from Schermaul et al's ESA-WVR work by amounts significantly larger than the errors associated with either study. We therefore decided to re-analyze this region, $10150-11190 \mathrm{~cm}^{-1}$, first. Results of this analysis should form the basis for a future re-investigation of the $8600-10150 \mathrm{~cm}^{-1}$ region. Figure 1 shows discrepancies at the $25 \%$ level for this longer wavelength region; previous studies of this region are less systematic than the work of Brown et $a l$.

Figure 2 compares the ESA-WVR and weak line data of Schermaul et al with the recent results of Coheur et al [17] for the region of the spectrum where the 

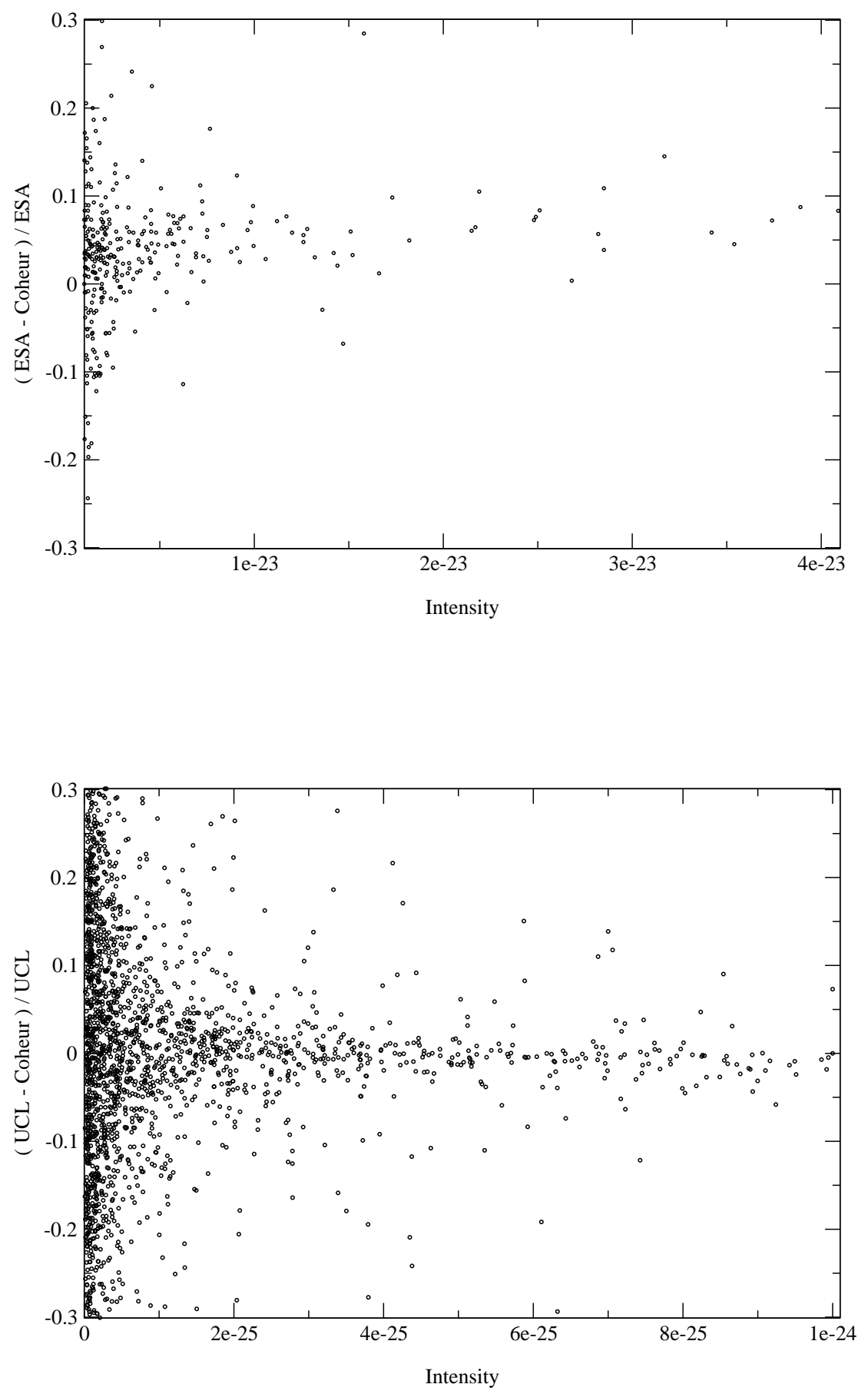

Fig. 2. A similar comparison of line intensities as in figure 1 but for the range $13000-15000 \mathrm{~cm}^{-1}$ and for different databases. Upper: relative differences for strong water vapor lines obtained from the ESA-WVR database [24] and Coheur et al [17] as a function of ESA-WVR line intensities. Lower: relative differences for weak water vapor lines from Schermaul et al (denoted UCL) [15] and Coheur et al [17] as a function of Schermaul et al line . 
respective studies overlap, which approximately covers the $4 \nu$ polyad. For the strong lines the ESA-WVR data [24] gives line intensities approximately $5 \%$ larger than Coheur et al, this increase is significantly less than the difference between ESA-WVR and HITRAN in the same frequency region. The weak line data of Schermaul et al [15] and Coheur et al [17] are actually in reasonable agreement. This latter comparison shows considerable scatter but this is probably just a reflection of the larger uncertainties in the measurements of these weaker features. A comparison of the weak line data of Schermaul et al [15], as analysed by us recently [28], with Brown et al [16] also shows reasonable agreement for the line intensities.

We identified four issues that need to be considered as part of a re-analysis of the ESA-WVR data: pressure in the cell, treatment of the baseline, effects due to the instrument function and parameterization of the functional form. We deal with each issue below. To address these issues we have refitted the spectra recorded by Schermaul et al [14] at $296 \mathrm{~K}$ and for pathlengths of $5 \mathrm{~m}, 32 \mathrm{~m}$ and $129 \mathrm{~m}$ in both water-air and pure water vapor. Schermaul et al did their fits using a line-fitting program called GREMLIN [43]. GREMLIN fits to four parameters: the line position $(\omega)$, peak height $(h)$, pressure broadening $(\gamma)$ and damping parameter $(D)$. Integration of the curve gives the line intensity, $I$. We performed fits using a new, interactive fitting program called GOBLIN [44]. Unless otherwise stated these fits used the same parameterization as GREMLIN against which GOBLIN was extensively tested.

Schermaul et al [14] employed both a pressure gauge and a humidity sensor to assess the water vapor content of their cell. They found that the instruments gave similar values for the water vapor content when the cell contained water vapor only. However, the partial pressure of water deduced from the pressure gauge for water-air mixtures was found to be significantly higher than that measured by the humidifier. Schermaul et al speculated that this effect could be due to water sticking to side of the tube at higher pressures, but the recent finding that microscopic droplets of water could remain in suspension regardless of pressure in the cell [45] could also provide an explanation for this behaviour.

Schermaul et al chose to use the value given by the humidifier. Their reasoning was that water sticking to the walls of the cell could not be taken into account correctly by the indirect pressure gauge measurement. Naturally the lower vapor pressure implied by the humidifier leads directly to higher line intensities. The difference is so large that while relying on the humidifier gives intensities systematically stronger than HITRAN, use of the pressure gauge leads to intensities systematically weaker than HITRAN by a similar amount.

As part of our re-analysis we compared strong line intensities obtained by fitting Schermaul et al's pure water vapor spectra with those obtained for 


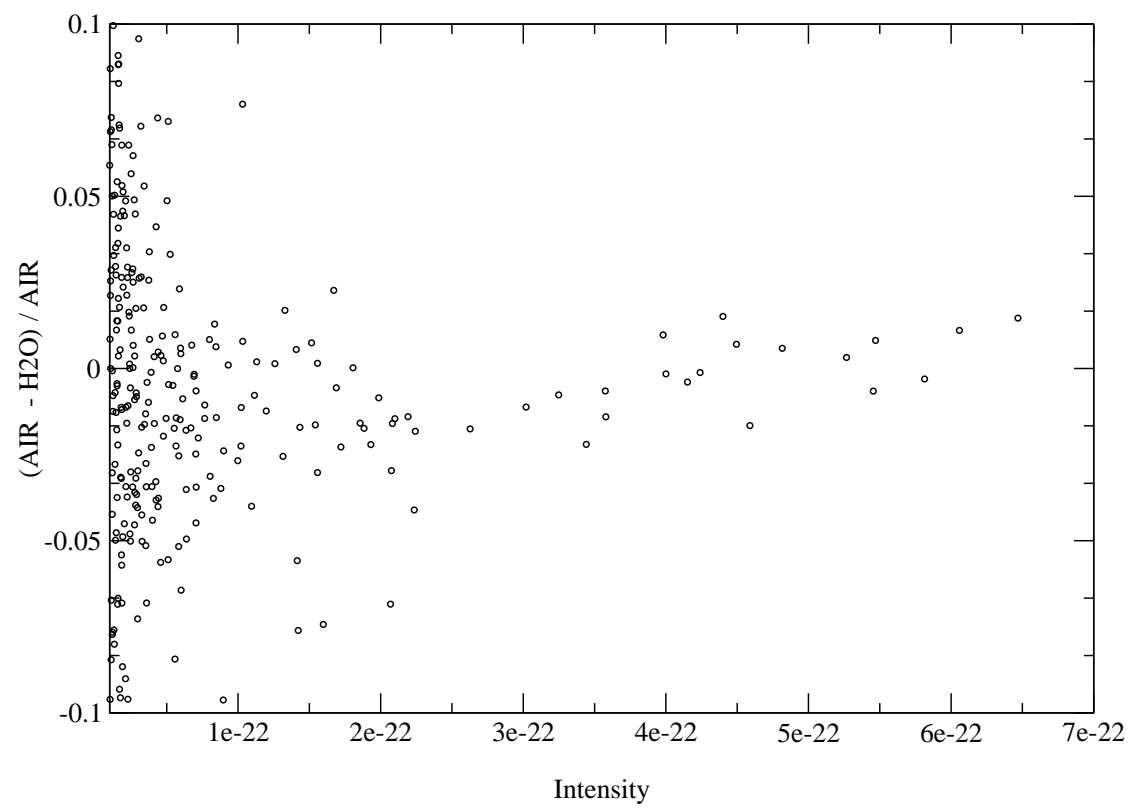

Fig. 3. Relative differences in line intensity as a function of line intensity (in $\mathrm{cm}$ molecules ${ }^{-1}$ ) for strong water vapor lines obtained by fitting the $296 \mathrm{~K}, 5 \mathrm{~m}$ water-air spectrum and the pure water vapor spectrum of Schermaul et al [14] in the range $10150-11190 \mathrm{~cm}^{-1}$.

the same pathlength in water-air. This comparison is best made for short pathlengths $(5 \mathrm{~m}, 32 \mathrm{~m})$ since the pure water spectrum saturates at longer paths. Figure 3 compares intensities fitted to a pure $5 \mathrm{~m}$ water vapor spectrum with those fitted to water-air spectra also obtained with a $5 \mathrm{~m}$ pathlength. The agreement for the strongest lines is reasonable. Conversely comparison of water-air spectra obtained at $5 \mathrm{~m}$ and $32 \mathrm{~m}$, Figure 4, show a systematic shift with the $32 \mathrm{~m}$ spectrum giving intensities approximately $5 \%$ stronger. It should be noted that the $5 \mathrm{~m}$ and $32 \mathrm{~m}$ spectra were recorded using quite different tubes.

The above analysis is completely based on the use of water partial pressures taken from the humidifier. Apart from potential uncertainties related both to this kind of pressure measurement as well as to the volume to surface ratio of the absorption cells used for different path lengths our tentative conclusion is that the humidifier readings do indeed represent the true partial pressure of water. Further analysis of problems with pressure determination must await resolution of problems to do with the baseline which we address next.

Water absorption spectra in the near infrared are very dense and, especially in regions where there are many strong lines, it is notoriously difficult to set 


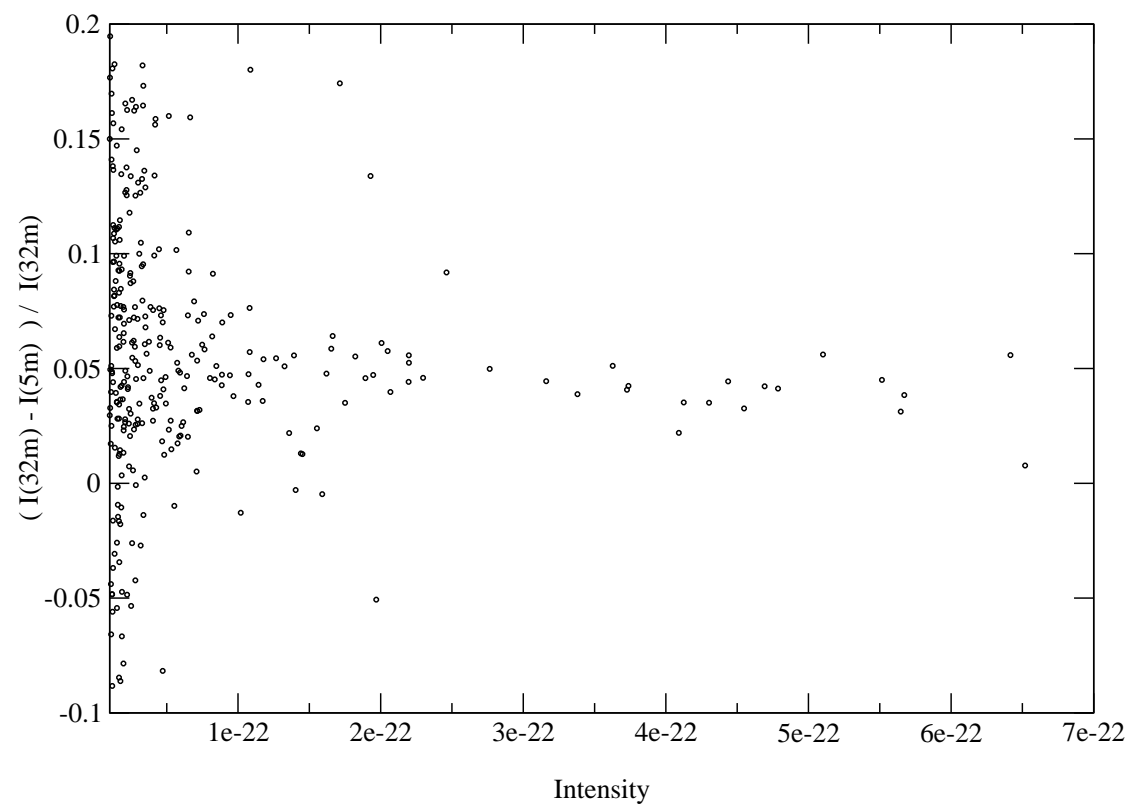

Fig. 4. Same as in figure 3 but for water vapor lines obtained from the $5 \mathrm{~m}$ and 32 $\mathrm{m}$ water-air spectra of Schermaul et al [14]

an unambiguous baseline. This is particularly true for water-air spectra where the strong lines are wide due to pressure broadening.

Schermaul et al determined their baseline in two steps [14]. First they divided the spectrum by the corresponding spectrum obtained using an empty cell. The baseline of the resulting raw spectrum was simulated by fitting polynomials to points were the baseline could indeed be determined. We checked this procedure by generating their spectra using the parameters from both ESAWVR [24] and HITRAN [2]. If the baseline determination was indeed done correctly then the function obtained by dividing the observed raw transmittance spectrum by the modeled transmittance spectrum should be a smooth curve. We did not find this in either case. Instead we found that using HITRAN parameters leads to a series of troughs in the baseline, see figure 5 . These troughs are intrinsically narrower than the line widths. Using the ESAWVR parameters leads to a series of much larger peaks in the baseline. These peaks are consistent with the intensity being overestimated. It would appear that the baseline correction has not been sufficiently well determined in these studies to eliminate all artifacts due to the baseline. We believe that the only way to make progress with this problem is to fit the spectra iteratively in such a fashion that a smooth baseline can be introduced during the fit, thereby taking into account all broadband experimental effects such as scattering due to microdroplets in suspension in the cell [45]. However before performing such a 

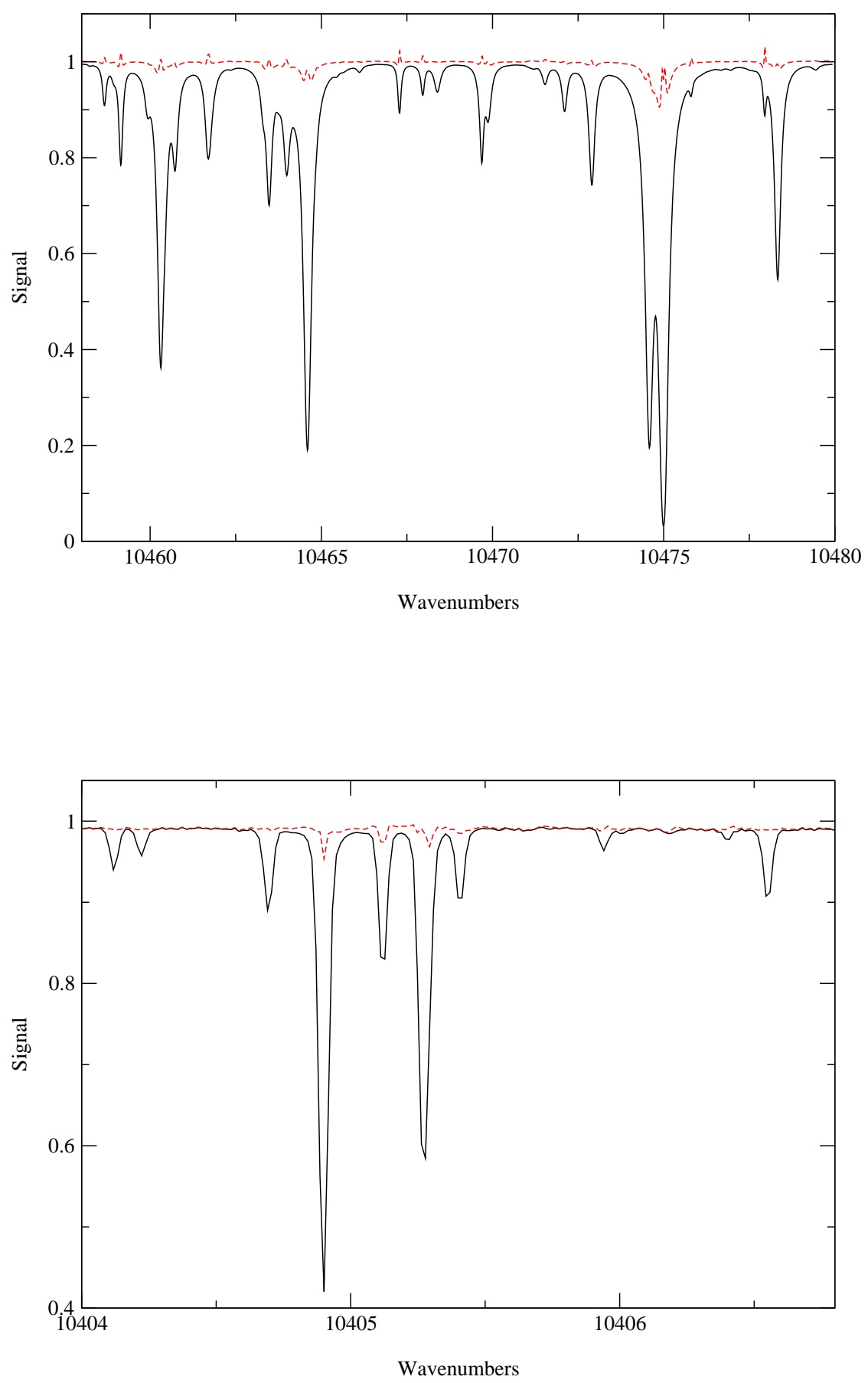

Fig. 5. Sample portions of the raw spectra recorded by Schermaul et al [14]. The upper plot shows a water-air spectrum, the lower plot a pure water vapor spectrum. Both spectra were recorded at $296 \mathrm{~K}$ with a pathlength of $129 \mathrm{~m}$. The dashed line is the baseline obtained by dividing the raw transmittance spectrum by a simulated spectrum generated using HITRAN [2] line parameters. 
fit it is necessary to determine the exact functional form of the line parameters to be used in the fit.

One issue that needs to be addressed is the precise effect of instrument function on the measured line profile [46]. Schermaul et al [14] performed tests on this and satisfied themselves that this function had little influence on their line profile. Our re-analysis of their data suggests the situation is less clear-cut. For example, Schermaul et al used a damping parameter, $D$ discussed below, to allow for collisional narrowing of their Doppler profile. Analysis of their $D$ values showed that in practice this parameter led to a Gaussian component of the Voigt profile which is systematically larger than the Doppler profile. One explanation for this that their analysis neglected line broadening effects due to the instrument profile. To test for a possible systematic effect from this on our fitted line intensities, we numerically integrated the area under a selection of lines. Such integration is only reliable where the line profile can be fully integrated, ie the full line can integrated including its wings and there are sufficient data points to properly characterize the lines. Under these circumstances the line intensities obtained by fitting and by integration agreed to about $1 \%$, which is the accuracy of the numerical integration. We also tested the effect on line intensities determined by fitting synthetic spectra convolved with an expected instrument profile. In this case uncertainties in the derived line intensities did not exceed $0.5 \%$. We are presently analyzing the possible instrumental effects on the line width parameters and results of this analysis will be reported elsewhere.

Brown et al [16] used three parameters $(\omega, h, \gamma)$ in their fits, allowing the measured temperature, $T$, to fix the damping ratio of the Voigt profile. Conversely Schermaul et al [14] argued that collision narrowing meant that the use of $T$ to determine the Doppler width of the line shape would lead to a fit which was not sufficiently flexible. The problem with this approach is that the fit does not give the temperature dependence of the line shape, or indeed, if collisional narrowing is significant, its pressure dependence. Problems with applying the ESA-WVR parameters have been exacerbated by the fact that the ESA-WVR data was made available in a HITRAN-style format [24] which means that the damping parameter, $D$, is not presented. This means that all spectral models based directly on the use of ESA-WVR are dubious as they use the wrong Voigt profile. It should be noted that Schermaul et al's models [23] used the correct line profile and that they performed many self-consistency checks using their line parameters which were found to reproduce a variety of their measured spectra to high accuracy.

To help understand these differences we have performed a number of fits using different line profiles. Table 1 summarizes these results for a sample selection of four lines which were chosen because they (a) covered a range of intensities, (b) were unblended as far as it is possible to tell and (c) lay in the region of the 
Table 1

Fitted water line intensities (and root mean square error for each fit normalized to peak height) in $\mathrm{cm}$ molecule ${ }^{-1}$, for four typical water lines and various spectra of Schermaul et al [14]. The spectra were all recorded at room temperature, with a pathlength of $l \mathrm{~m}$. Fits used either $n=3,(\omega, h, \gamma)$, or $n=4,(\omega, h, \gamma, D)$ parameters. A null entry corresponds to an experimental set-up for which the line were found to be saturated. The corresponding intensities from HITRAN $[2,16]$ and ESA-WVR $[23,24]$ are given for comparison. The error quoted is that due to noise, other sources of error are discussed in the text.

\begin{tabular}{|c|c|c|c|c|c|c|c|c|c|c|}
\hline Sample & $l$ & $n$ & $\begin{array}{l}10168.14 \\
I \times 10^{-24} \\
\end{array}$ & $\begin{array}{c}\text { error } \\
\% \\
\end{array}$ & $\begin{array}{l}10713.49 \\
I \times 10^{-23} \\
\end{array}$ & $\begin{array}{c}\text { error } \\
\% \\
\end{array}$ & $\begin{array}{l}10610.74 \\
I \times 10^{-22} \\
\end{array}$ & $\begin{array}{c}\text { error } \\
\% \\
\end{array}$ & $\begin{array}{l}10600.85 \\
I \times 10^{-22} \\
\end{array}$ & $\begin{array}{c}\text { error } \\
\%\end{array}$ \\
\hline $\mathrm{H}_{2} \mathrm{O}$ & 5 & 4 & 8.63 & 1.6 & 6.59 & 0.5 & 1.04 & 0.7 & 1.97 & 0.3 \\
\hline $\mathrm{H}_{2} \mathrm{O}$ & 5 & 3 & 8.98 & 1.9 & 6.73 & 0.7 & 1.06 & 0.9 & 2.00 & 0.4 \\
\hline $\mathrm{H}_{2} \mathrm{O}+\mathrm{air}$ & 5 & 4 & 9.00 & 5.0 & 6.44 & 0.8 & 1.03 & 0.4 & 1.93 & 0.4 \\
\hline $\mathrm{H}_{2} \mathrm{O}+\mathrm{air}$ & 5 & 3 & 9.60 & 4.8 & 6.42 & 0.8 & 1.02 & 0.5 & 1.92 & 0.4 \\
\hline $\mathrm{H}_{2} \mathrm{O}$ & 32 & 4 & 9.21 & 0.4 & & & & & & \\
\hline $\mathrm{H}_{2} \mathrm{O}$ & 32 & 3 & 9.13 & 0.5 & & & & & & \\
\hline $\mathrm{H}_{2} \mathrm{O}+\mathrm{air}$ & 32 & 4 & 9.54 & 0.5 & 6.90 & 0.2 & 1.08 & 0.1 & 2.05 & 0.2 \\
\hline $\mathrm{H}_{2} \mathrm{O}+\mathrm{air}$ & 32 & 3 & 9.41 & 0.5 & 6.83 & 0.3 & 1.07 & 0.2 & 2.03 & 0.3 \\
\hline $\mathrm{H}_{2} \mathrm{O}$ & 129 & 4 & 9.30 & 0.2 & & & & & & \\
\hline $\mathrm{H}_{2} \mathrm{O}$ & 129 & 3 & 9.18 & 0.4 & & & & & & \\
\hline $\mathrm{H}_{2} \mathrm{O}+\mathrm{air}$ & 129 & 4 & 9.49 & 0.1 & 6.91 & 0.2 & 1.07 & 0.3 & 2.04 & 0.2 \\
\hline $\mathrm{H}_{2} \mathrm{O}+\mathrm{air}$ & 129 & 3 & 9.23 & 0.2 & 6.81 & 0.3 & 1.08 & 0.3 & 2.02 & 0.3 \\
\hline \multicolumn{2}{|l|}{ ESA-WVR } & \multicolumn{3}{|c|}{9.57} & \multicolumn{2}{|l|}{6.92} & \multicolumn{2}{|l|}{1.09} & \multicolumn{2}{|l|}{2.09} \\
\hline \multicolumn{2}{|l|}{ HITRAN } & \multicolumn{3}{|c|}{8.98} & \multicolumn{2}{|l|}{6.49} & \multicolumn{2}{|l|}{1.01} & \multicolumn{2}{|l|}{1.93} \\
\hline \multicolumn{2}{|c|}{ assignments } & \multicolumn{3}{|c|}{ (121) $6_{15}-7_{16}$} & \multicolumn{2}{|c|}{$(300) 5_{24}-4_{13}$} & \multicolumn{2}{|c|}{$(201) 4_{22}-4_{23}$} & \multicolumn{2}{|c|}{ (201) $2_{21}-2_{20}$} \\
\hline
\end{tabular}

spectrum analyzed by Brown et al [16]. Besides the integrated line intensity, $I$, Table 1 also gives an error estimate. This percentage error is the root mean square error for each fit divided by the peak height; it represents the lower limit of the error but is indicative of the quality of the fit.

As can be seen, the fits show significant variation in the line intensity according to which spectrum was fitted. In particular our new fits give intensities which are lower than those of ESA-WVR but still above those given in HITRAN. The fits which use three or four parameters to represent the same data give 
very similar results, suggesting that three parameters and a pre-determined temperature are indeed sufficient. However it should be noted that both three and four parameter fits show pronounced systematic errors when they are used to reconstruct the baseline in the manner of figure 3. A full discrimination between the two fitting procedures must therefore await resolution of the problem with the baseline.

\section{Conclusions}

Although the HITRAN data base is an invaluable source of data on water line parameters, there remains a number of outstanding problems with these parameters at near infrared and visible wavelengths. Our strategy for tackling these issues is based on four separate approaches: analysis of water-air spectra to determine parameters for strong lines, analysis of long pathlength pure water spectra to obtain parameters for weaker water lines, analysis of isotopically enhanced spectra to obtain data on water isotopomers and the use of high-level theoretical calculations not only to provide line assignments but also help determine line intensities for both observed and missing lines.

We have shown that the determination of strong line intensities for water vapor is particularly difficult. This is corroborated by the results of studies by Schermaul and co-workers $[14,19,23]$ who have suggested that water line intensities in the near infrared and red should be stronger than those given in HITRAN and partially supported by the results by Brown et al [16] in the near infrared, (and included in the recent addition of HITRAN [2]) which give lower intensities than the work of Schermaul and co-workers. Our partial re-analysis of the Schermaul et al data considers a number of reasons for this and suggests that a better determination of the baseline is required. It would appear that a thorough re-analysis is likely to lead to intensities closer to those determined by Brown et al. However some differences with HITRAN in other portions of the spectrum, particularly in the $8600-10150 \mathrm{~cm}^{-1}$ region, are likely to remain. This re-analysis, which will involve simultaneous fits to all the spectra recorded by Schermaul et al and iterative determination of the baseline, is currently underway.

Fitting spectra is of course an important task but it is also necessary to demonstrate that the line parameters so determined are the correct ones. The easiest procedure for this is to check for self-consistency by using the parameters to model the spectrum that has been fitted. The problem with this procedure is that any systematic errors are often self-cancelling and thus remain hidden. Alternative means of validating line parameters for difficult species such as water are therefore essential. 
There are a number of compelling reasons for compiling databases of water transitions apart from improving the accuracy of climate model predictions. These include the retrieval of atmospheric water vapor densities from satellite platforms and the simulations of high resolution atmospheric spectra from ground-based instrumentation. Such high resolution spectra may also be used to help test spectral databases, although we note that these spectra often have their own problems, for instance due to uncertainties in the atmospheric water vapor profile involved (see [47] for example). Several studies have used high resolution, ground-based, atmospheric spectra to test databases of water transitions [48-50] and more recently tests using satellite data have been attempted [51]. Thus controlled situations in which the atmospheric water vapor column is sufficiently well-determined from independent water vapor measurements can be used to systematically validate our water line parameters using atmosphere measurements, a methodology we are currently pursuing.

\section{Acknowledgement}

We thank Jim Brault and Roland Schermaul for helpful conversations during the course of this work. We thank a referee for helpful comments on our original manuscript. This work has been supported by the UK Research Councils NERC and EPSRC, The Royal Society, the INTAS foundation and the Russian Fund for Fundamental Studies. 


\section{References}

[1] Rothman LS, Rinsland CP, Goldman A, Massie ST, Edwards D, Flaud JM, Perrin A, Camy-Peyret C, Dana V, Mandin JY, Schroeder J, McCann A, Gamache RR, Wattson R, Yoshino K, Chance K, Jucks K, Brown L, Nemtchinov V, Varanasi P. The hitran molecular spectroscopic database and hawks (hitran atmospheric workstation): 1996 edition, J. Quant. Spec. Radiative Transf. 1998;60:665-710.

[2] Rothman LS, HITRAN 2000, www.hitran.com.

[3] Camy-Peyret C, Flaud JM, Mandin JY, Chevillard JP, Brault J, Ramsay DA, Vervloet M, Chauville J. The high-resolution spectrum of water-vapor between 16500 and $25250 \mathrm{~cm}^{-1}$, J. Mol. Spectrosc. 1985;113:208-228.

[4] Mandin JY, Chevillard JP, Camy-Peyret C, Flaud JM. The high-resolution spectrum of water vapor between 13200 and $16500 \mathrm{~cm}^{-1}$, J. Mol. Spectrosc. 1986;116:167-190.

[5] Chevilliard JP, Mandin JY, Flaud JM, Camy-Peyret C. $\mathrm{H}_{2}{ }^{18} \mathrm{O}$ - The (030), (110), and (011) interacting states - line positions and intensities for the $3 v_{2}$, $v_{1}+v_{2}$ and $v_{2}+v_{3}$ bands, Can. J. Phys. 1985;63:1112-1127.

[6] Chevilliard JP, Mandin JY, Flaud JM, Camy-Peyret C. $\mathrm{H}_{2}{ }^{1} 6 \mathrm{O}$ : line positions and intensities between 9500 and $11500 \mathrm{~cm}^{-1}$. The interacting vibrational states (041), (220), (121), (022), (300), (201), (102) and (003), Can. J. Phys. $1989 ; 67: 1065-1084$.

[7] Toth RA, Extensive measurements of $\mathrm{H}_{2}{ }^{16} \mathrm{O}$ line frequencies and strengths 5750 to $7965 \mathrm{~cm}^{-1}$, Applied Optics 1994;33:4851-4867.

[8] Flaud JM, Camy-Peyret C, Bykov A, Naumenko O, Petrova T, Scherbakov A, Sinitsa L. The high-resolution spectrum of water vapor between 11600 and $12750 \mathrm{~cm}^{-1}$, J. Mol. Spectrosc. 1997;183:300-309.

[9] Flaud JM, Camy-Peyret C, Bykov A, Naumenko O, Petrova T, Scherbakov A, Sinitsa L. The water vapor linestrengths between 11600 and $12750 \mathrm{~cm}^{-1}, \mathrm{~J}$. Mol. Spectrosc. 1997;185:211-221.

[10] Hu SM, Ulenikov ON, Onopenko GA, Bekhtereva ES, He SG, Wang XH, Lin $\mathrm{H}$, Zhu QS. High-Resolution Study of the $\left(v_{1}+v_{2}+v_{3}=3\right)$ Polyad of Strongly Interacting Vibrational Bands of $\mathrm{D}_{2} \mathrm{O}$, J. Mol. Spectrosc. 2000;203:228-234.

[11] Ulenikov ON, Hu SM, Bekhtereva ES, Onopenko GA, Wang XH, He SG, Zheng JJ, Zhu QS. High-Resolution Fourier Transform Spectrum of HDO in the Region 6140-7040 $\mathrm{cm}^{-1}$, J. Mol. Spectrosc. 2001;208:224-235.

[12] Carleer M, Jenouvrier A, Vandaele AC, Bernath PF, Marienne MF, Colin R, Zobov NF, Polyansky OL, Tennyson J, Savin VA. The near infrared, optical and near ultraviolet overtone spectrum of water, J. Chem. Phys. 1999;111:24442450 . 
[13] Zobov NF, Belmiloud D, Polyansky OL, Tennyson, J, Shirin SV, Carleer M, Jenouvrier A, Vandaele AC, Bernath PF, Marienne MF, Colin R. The near ultraviolet rotation-vibration spectrum of water, J. Chem. Phys. 2000;113:15461552 .

[14] Schermaul R, Learner RCM, Newnham DA, Williams RG, Ballard J, Zobov NF, Belmiloud D, Tennyson J. The water vapour spectrum in the region $8600-15000 \mathrm{~cm}^{-1}$ : experimental and theoretical studies for a new spectral line database i: Laboratory measurements, J. Molec. Spectrosc. 2001;208:32-42.

[15] Schermaul R, Brault JW, Canas AAD, Learner RCM, Polyansky OL, Zobov $\mathrm{NF}$, Belmiloud $\mathrm{D}$, Tennyson J. Weak line water vapour spectrum in the regions $13200-15000 \mathrm{~cm}^{-1}$, J. Molec. Spectrosc. 2002;211:169-178.

[16] Brown LR, Toth RA, Dulick M. Empirical Line Parameters of $\mathrm{H}_{2}{ }^{16} \mathrm{O}$ near 0.94 $\mu \mathrm{m}$ : Positions, Intensities and Air-Broadening Coefficients, J. Mol. Spectrosc. $2002 ; 212: 57-82$.

[17] Coheur PF, Fally S, Carleer M, Clerbaux C, Colin R, Jenouvrier A, Mérienne MF, Hermanns C, Vandaele AC. New water vapor line parameters in the 26000$13000 \mathrm{~cm}^{-1}$ region, J. Quant. Spec. Radiat. Transf. 2002;74:493-510.

[18] Naumenko O, Campargue A, High-order resonance interactions in HDO: Analysis of the absorption spectrum in the $14980-15350 \mathrm{~cm}^{-1}$ spectral region, J. Mol. Spectrosc. 2000;199:59-72.

[19] Belmiloud D, Schermaul R, Smith K, Zobov NF, Brault J, Learner RCM, Newnham DA, Tennyson J. New studies of the visible and near infra-red absorption by water vapour and some problems with the database, Geophys. Res. Lett. 2000;27:3703-3706.

[20] Zhong W, Haigh JD, Belmiloud D, Schermaul R, Tennyson J. The impact of new water vapour spectral line paramaters on the calculation of atmospheric absorption, Quart. J. Royal Meteorol. Soc. 2001;127:1615-1626.

[21] Zhong W, Haigh JD, Belmiloud D, Schermaul R, Tennyson J. Note on 'The impact of new water vapour spectral line paramaters on the calculation of atmospheric absorption', Quart. J. Royal Meteorol. Soc. 2002;128:1387-1388.

[22] Giver LP, Chackerian Jr. C, Varanasi P. Visible and near-infrared (H2O)-O16 line intensity corrections for HITRAN-96, J. Quant. Spec. Radiat. Transf. 2000;66:101-105.

[23] Schermaul R, Learner RCM, Newnham DA, Ballard J, Zobov NF, Belmiloud $\mathrm{D}$, Tennyson J. The water vapour spectrum in the region $8600-15000 \mathrm{~cm}^{-1}$ : experimental and theoretical studies for a new spectral line database ii: Construction and validation, J. Molec. Spectrosc. 2001;208:43-50.

[24] Schermaul R, Zobov, NF, Learner RCM, Newnham DA, Ballard J, Tennyson J. ESA-WVR, http://badc.nerc.ac.uk/data/esa-wv/. 
[25] Partridge H, Schwenke DW. The determination of an accurate isotope dependent potential energy surface for water from extensive ab initio calculations and experimental data, J. Chem. Phys. 1997;106:4639.

[26] Callegari A, Theule P, Tolchenov RN, Zobov NF, Polyansky OL, Tennyson, J, Muenter JS, Rizzo TR. Dipole moments of highly vibrationally excited water, Science 2002;297:993-995.

[27] Tolchenov RN, Tennyson J, Brault JW, Canas AAD, Schermaul R. Weak line water vapor spectrum in the $11,787-13,554 \mathrm{~cm}^{-1}$ region, J. Molec. Spectrosc. $2002 ; 215: 269-274$.

[28] Tolchenov RN, Tennyson J, Zobov NF, Shirin SV, Polyansky OL, Maurellis AN, Weak line water vapor spectrum in the $9.200-12,800 \mathrm{~cm}^{-1}$ region, J. Molec. Spectrosc. (to be submitted).

[29] Naus H, Ubachs W, Levert PF, Polyansky OL, Zobov NF, Tennyson J. Cavityring-down spectroscopy on water vapor in the range 555-604 nm, J. Molec. Spectrosc. 2001;205:117-121.

[30] Learner RCM, Zhong W, Haigh JD, Belmiloud D, Clarke J. The contribution of unknown weak water vapor lines to the absorption of solar radiation, Geophys.Res.Lett. 1999;26:3609-3612.

[31] Chevilliard JP, Mandin JY, Flaud JM, Camy-Peyret C. The first hexad [(040), (120), (021), (200), (101), (002)] of $\mathrm{H}_{2}{ }^{18} \mathrm{O}$ - experimental energy-levels and line-intensities, Can. J. Phys. 1986;64:746-761.

[32] Chevilliard JP, Mandin JY, Flaud JM, Camy-Peyret C. $\mathrm{H}_{2}{ }^{18} \mathrm{O}-$ line positions and intensities between 9500 and $11500 \mathrm{~cm}^{-1}$ - the (041), (220), 121), (300), (201), (102), and (003) interacting states, Can. J. Phys. 1987;65:777-789.

[33] Bykov A, Naumenko O, Petrova T, Scherbakov A, Sinitsa L, Mandin JY, CamyPeyret C, Flaud JM. The second decade of $\mathrm{H}_{2}{ }^{18} \mathrm{O}$ - line positions and energylevels, J. Mol. Spectrosc. 1995;172:243-253.

[34] Tanaka M, Brault JW, Tennyson J. Absorption spectrum of $\mathrm{H}_{2}{ }^{18} \mathrm{O}$ in the 12,400 - $14520 \mathrm{~cm}^{-1}$ range, J. Molec. Spectrosc. 2002;216:77-80.

[35] Tanaka M, Brault JW, Tennyson J, Absorption spectrum of $\mathrm{H}_{2}{ }^{17} \mathrm{O}$ in the 11,300 - $14520 \mathrm{~cm}^{-1}$ range, J. Molec. Spectrosc. (to be submitted).

[36] Polyansky OL, Zobov NF, Viti S, Tennyson J, Bernath PF, Wallace L, Water in the sun: line assignments based on variational calculations, Science 1977;277:346-349.

[37] Polyansky OL, Császár AG, Shirin SV, Zobov NF, Barletta P, Tennyson J, Schwenke DW, Knowles PJ. High accuracy ab initio rotation-vibration transitions of water, Science 2003;299:539-542.

[38] Schwenke DW, New $\mathrm{H}_{2} \mathrm{O}$ rovibrational line assignments, J. Mol. Spectrosc. 1998;190:397-402. 
[39] Polyansky OL, Zobov NF, Viti S, Tennyson J. Water vapour line assignments in the near infrared, J. Mol. Spectrosc. 1998;189:291-300.

[40] Jenouvrier A, Marienne MF, Carleer M, Colin R, Vandaele AC, Bernath PF, Polyansky OL, Tennyson J. The visible and near ultraviolet overtone spectrum of HOD, J. Molec. Spectrosc. 2001;209:165-168.

[41] Schwenke DW, Partridge H. Convergence testing of the analytic representation of an ab initio dipole moment function for water: Improved fitting yields improved intensities, J. Chem. Phys. 2000;113:6592-6597.

[42] Lynas-Gray AE, et al, A high accuracy ab initio dipole surface for water, J. Chem. Phys. (to be submitted).

[43] Brault JW, Program GREMLIN (unpublished).

[44] Tolchenov RN, Program GOBLIN (to be published).

[45] Carleer M, Kiseleva M, Fally S, Coheur PF, Clerbaux C, Colin R, Daumont L, Jenouvrier A, Mérienne MF, Hermanns C, Vandaele AC. Laboratory Fourier transform spectroscopy of the water absorption continuum from 2500 to 22500 $\mathrm{cm}^{-1}$, in: Weakly Interacting Molecular Pairs: Unconventional Absorbers of Radiation in the Atmosphere, NATO Science Series IV, Kluwer, Dordrecht, 2003 in press.

[46] Davis SP, Abrams MC, Brault JW. Fourier Transform Spectrometry, Academic, New York, 2001.

[47] Lang R, Maurellis AN, van der Zande WJ, Aben I, Landgraf J, Ubachs W. Forward modeling and retrieval of water vapor from the global ozone monitoring experiment: Treatment of narrowband absorption spectra, J. Geophys. Res. 2002;D107 art. no. 4300.

[48] Harder JW, Brault JW. Atmospheric measurements of water vapor in the 442nm region. J. Geophys. Res. 1997;D105:6245-6252.

[49] Smith KM, Newnham DA. High-resolution atmospheric absorption by water vapor in the 830-985 $\mathrm{nm}$ region: Evaluation of spectroscopic databases. Geophys. Res. Lett. 2001;28:7541-7552.

[50] Veihelmann B, Lang R, Smith KM, Newnham DA, van der Zande WJ. Evaluation of spectroscopic databases of water vapor between 585 and $600 \mathrm{~nm}$. Geophys. Res. Lett. 2002;29 art. no. 1752 .

[51] Maurellis AN, Lang R, Williams JE, van der Zande WJ, Smith K, Tennyson $\mathrm{J}$, Tolchenov RN. The impact of new water vapor spectroscopy on satellite retrievals, in: Weakly Interacting Molecular Pairs: Unconventional Absorbers of Radiation in the Atmosphere, NATO science series IV, Kluwer, Dordrecht, 2003 , in press. 